\title{
The International Collaboration to Improve Respiratory Health in Children (INCIRCLE) ERS Clinical Research Collaboration
}

\author{
Peter D. Sly ${ }^{1}$ and Zoltán Hantos ${ }^{2}$ on behalf of the INCIRCLE Clinical Research \\ Collaboration
}

Affiliations: ${ }^{1}$ Children's Health and Environment Program, Child Health Research Centre, The University of Queensland, Brisbane, Australia. ${ }^{2}$ Dept of Anaesthesiology and Intensive Therapy, Semmelweis University, Budapest, Hungary.

Correspondence: Peter D. Sly, Children's Health and Environment Program, Child Health Research Centre, The University of Queensland, 62 Graham Street, South Brisbane, QLD 4101, Australia. E-mail: p.slyquq.edu.au

@ERSpublications

The INCIRCLE ERS Clinical Research Collaboration has made great advances in developing new modalities of oscillometry for use in infants and young children, and this has evoked interest from the lung function device industry http://ow.ly/FPYz30mC3La

Cite this article as: Sly PD, Hantos Z. The International Collaboration to Improve Respiratory Health in Children (INCIRCLE) ERS Clinical Research Collaboration. Eur Respir J 2018; 52: 1801867 [https://doi.org/ 10.1183/13993003.01867-2018].

\section{Rationale}

Respiratory disease is a major component of the global burden of disease, with a reduction in deaths from childhood pneumonia but an increase in chronic respiratory disease over the past few decades [1-3]. There is an increasing recognition that low lung function poses a major risk for acute and chronic lung disease. Lung function tracks closely along centiles throughout childhood $[4,5]$, with lung function at birth determining lung function in later life [6]. Childhood asthma is associated with low lung function [7, 8], with the deficit seen on the first occasion that it is measured. Low lung function in childhood, with failure to reach predicted peak lung function in early adult life, is a major risk factor for chronic obstructive pulmonary disease (COPD), responsible for about $50 \%$ of cases in adults $[9,10]$.

Airway obstruction, generally indicated by a ratio of the forced expiratory volume in $1 \mathrm{~s}$ (FEV1) to the forced vital capacity (FVC) of $<0.7$ following bronchodilator inhalation, is a hallmark of COPD [11]. Indeed, the reliance on forced spirometry (so named to indicate the forced expiration required) may be one of the reasons that the major impact of early-life factors on COPD has been under-recognised [6]. Forced spirometry can be challenging in young children [12], with reported success rates varying from $30 \%$ to $>80 \%[12,13]$. Young children find several aspects of spirometry difficult, including: taking a full breath in to total lung capacity; achieving a rapid onset of forced expiration; blowing hard enough to achieve expiratory flow limitation; and maintaining forced expiration to residual volume [12]. Given these difficulties, it is easy to understand why FVC may be underestimated in young children. In addition, in healthy young children, FEV1 approximates FVC, with a FEV1/FVC ratio of about $94 \%$ in children aged 5-7 years [14]. Thus, the physiological meaning of FEV1 differs in young children. This, together with the 
likely underestimation of FVC, means detecting airway obstruction by forced spirometry is challenging in young children. A better approach is required.

\section{The International Collaboration to Improve Respiratory Health in Children}

The International Collaboration to Improve Respiratory Health in Children (INCIRCLE) European Respiratory Society (ERS) Clinical Research Collaboration (CRC) was supported by the ERS Paediatrics Assembly and initially funded in the first round of such collaborations in 2014 [15]. In the initial application we proposed to form an international collaborative group to develop intra-breath tracking or temporal oscillometry (T-oscillometry), a novel adaptation of conventional oscillometry that made estimates of respiratory system impedance $(\mathrm{Zrs})$ at multiple points during tidal breathing. Note that the term "forced" has been deleted as no forced expiration is involved. T-oscillometry demonstrates changes in $Z$ rs as lung volume and respiratory flow changes during the normal breathing process. One of the motives behind this initiative was the realisation that, while oscillometry was easy for young children to perform, its conventional modality was better suited to showing differences between groups of subjects than to determining which children had abnormal lung function [12, 16-18]. The initial collaboration included groups experienced in lung function testing in young children in Australia, Brazil, Hungary, Italy, South Africa, the UK and the USA. The previous CRC conditions restricted applications to 10 chief investigators, which limited the number of centres we could include. The INCIRCLE aims included: development and characterisation of T-oscillometry for use in infants and young children; determination of the clinical utility of T-oscillometry in a variety of respiratory conditions; creation of normative values that would allow reporting of T-oscillometry outcome variables as standard deviation (Z) scores; and facilitation of commercial availability of T-oscillometry beyond specialist centres. When the INCIRCLE CRC was re-funded in 2017 for a further 3-year period, it was expanded to include more centres.

\section{Improved understanding of T-oscillometry outcome variables}

One of the barriers to more widespread use of oscillometry by clinicians is the engineering-based terminology used. Physicians get confused by the various terms used to describe the components of $Z_{\text {rs }}$, including resistance $(R)$ and reactance $(X)$, and even more by the real and imaginary parts, the in-phase and out-of-phase components. The understanding of $R$ is reasonable but confusion reigns over the interpretation of $X$. Clinicians are not used to negative numbers as test results and confusion increases when a more negative, i.e. larger-magnitude negative number, indicates a worse result. In an attempt to provide simplified results, we have advocated fitting a "resistance-inertance-compliance" $(R L C)$ model to the $R$ and $X$ spectra (figure 1 ). The model is described as:

$$
Z_{\mathrm{rs}}=R+\mathrm{j} \omega L-(1 / \mathrm{j} \omega C)
$$

where $\mathrm{j}$ is the imaginary unit and $\omega$ represents angular frequency.

This allows reporting for a single number of $R$, which represents the plateau value $(10-20 \mathrm{~Hz})$ seen in the $R$-frequency plot and provides an estimate of Newtonian $R$ of the respiratory system mainly reflecting

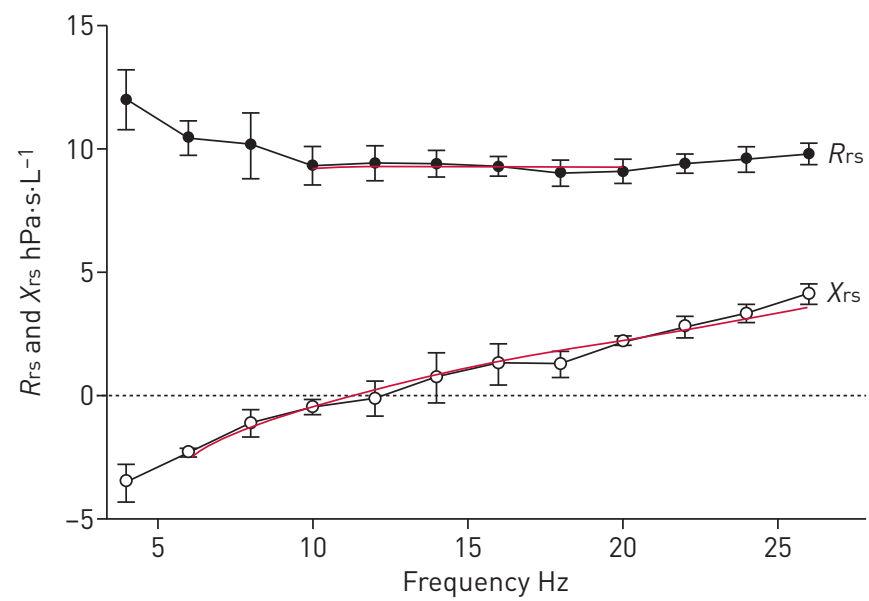

FIGURE 1 Fitting a model to resistance and reactance spectra measured in a preschool-aged child to provide estimates of respiratory resistance and compliance. The data points (mean and sD) represent the average of all technically acceptable replicate measurements and red lines indicate the model fit to the data points over specified ranges of frequency. $R$ rs: respiratory system resistance; $X_{r s}$ : respiratory system reactance. 
airway $R$. Similarly, the curve fitted to the $X$-frequency plot $(6-26 \mathrm{~Hz})$ provides an estimate of respiratory compliance $(C)$ and inertance $(L)$, the latter determining $X$ at higher frequencies. These single numbers are more easily understood, although the consistency of the $R L C$ model weakens in disease. Whether the use of the RLC model facilitates the use of oscillometry remains to be seen. We have demonstrated the changes occurring in $R$ and $C$ in the first 3 days of life in healthy neonates [19], shown the ability of $C$ to distinguish between infants born to mothers who smoked during pregnancy and those born to nonsmoking mothers [20] and provided normative data for preschool-aged children [21].

\section{T-oscillometry}

Knowledge that the respiratory system is not linear and that both volume and flow influence respiratory mechanics is not new. However, many lung function techniques ignore this. This is especially true for conventional oscillometry, where $Z_{r s}$ is averaged over several breathing cycles to produce single estimates for $R$ and $X$ at each frequency contained in the driving signal. T-oscillometry uses a single-frequency (sinusoidal) test signal $(16 \mathrm{~Hz}$ for infants, $10 \mathrm{~Hz}$ for children and $8 \mathrm{~Hz}$ for adults) and makes a series of estimates of $R$ and $X$ during tidal breathing (figure 2). Volume dependence is calculated from $R$ and $X$ at points of zero flow occurring at the end of inspiration and expiration (figure $2 a$ ) and flow dependence can be described by points of maximal inspiratory and expiratory flow (figure 2a). The changes in $R$ and $X$ during tidal breathing plotted as a function of tidal volume are obvious (figure $2 \mathrm{~b}$ ) and clearly demonstrate the problem with averaging over several breathing cycles. The volume dependence is quantified by comparing the values of $R$ or $X$ at end-inspiration and end-expiration, shown for $\Delta R$ in figure $2 c$.

\section{Oscillometry in unsedated infants during natural sleep}

The INCIRCLE site in Cape Town (South Africa) has used both conventional (spectral) oscillometry and T-oscillometry to measure lung function in the longitudinal cohort study, the Drakenstein Child Health Study. Lung function was measured at 5-10 weeks, 1 year and 2 years of age in unsedated infants. The feasibility at 5-10 weeks of age was outstanding, with measurements successful in $>80 \%$ of infants $[20,22]$. This population is being followed up in the preschool years.
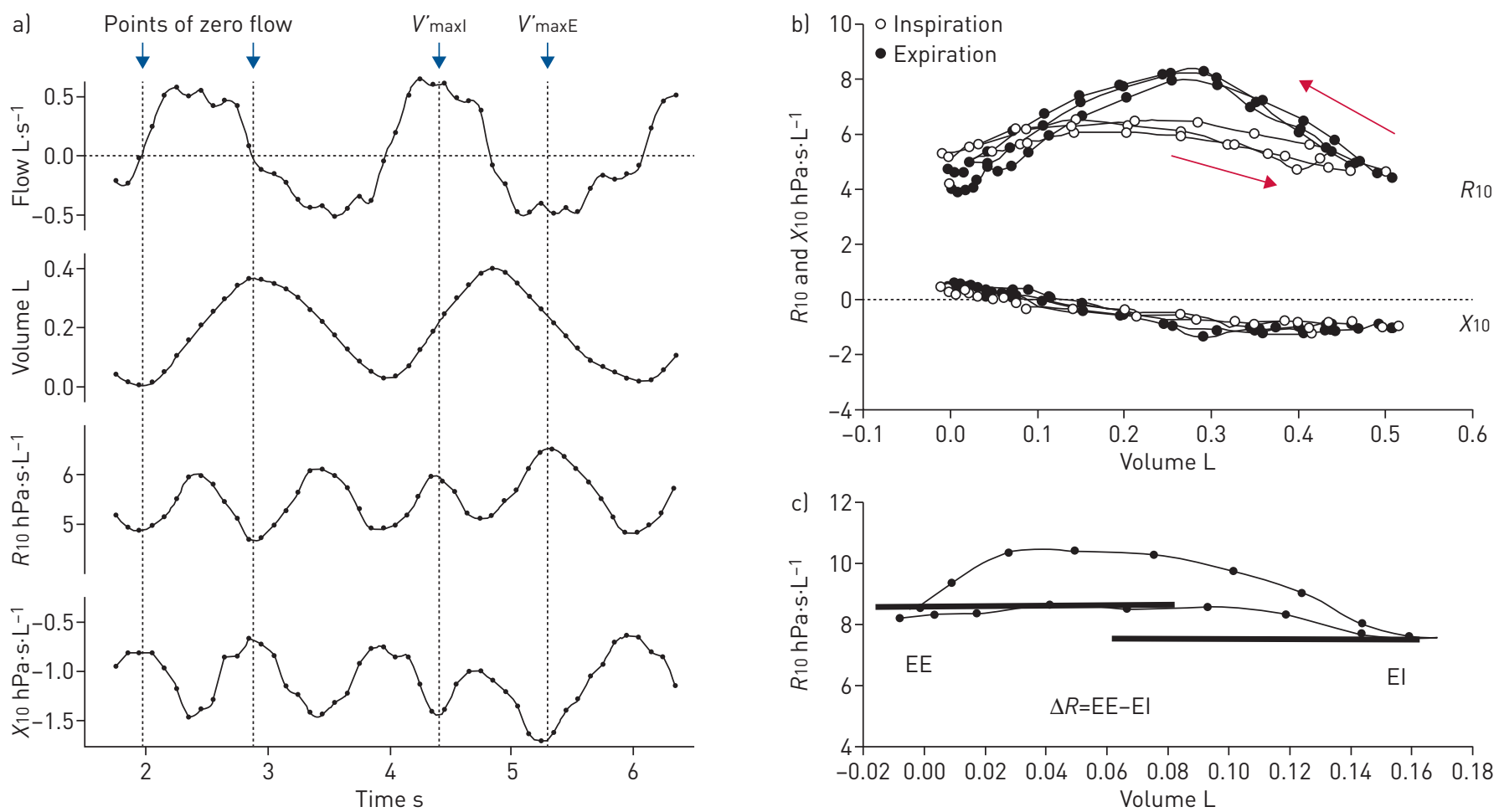

FIGURE 2 Schematic representation of temporal oscillometry (T-oscillometry) measured at $10 \mathrm{~Hz}$ in a healthy preschool-aged child. a) The changes in resistance $(R)$ and reactance $(X)$ at $10 \mathrm{~Hz}\left(R 10\right.$ and $X_{10}$, respectively) during tidal breathing. $V^{\prime}$ maxl: maximal inspiratory flow; $V^{\prime}$ maxE: maximal expiratory flow. b) The changes in $R 10$ and $X_{10}$ as a function of volume; the direction of breathing (inspiration and expiration) is shown by the arrows. c) Calculation of $\Delta R$ to quantify the volume dependence of $R 10$. EE: end-expiration; El: end-inspiration. 
Oscillometry in preschool-aged children

We have demonstrated the feasibility and utility of measuring T-oscillometry in preschool-aged children $[23,24]$. Normative data have been collected by measuring children in a variety of settings, including in kindergartens [24]. Measurements have also been made in children with acute wheeze in the emergency department and during recovery phases of acute asthma exacerbations [23]. Under these conditions, feasibility remains excellent with $80-90 \%$ of children able to perform the test and produce good quality data. We have shown that the volume dependence of $\Delta R$ was a highly sensitive and specific measure of airway obstruction in wheezy children, with a cut-off value of $1.42 \mathrm{hPa} \cdot \mathrm{s} \cdot \mathrm{L}^{-1}$, giving an area under the receiver operating characteristic curve of 0.95 [23]. T-oscillometry was also useful in demonstrating a bronchodilator response, with the value of $\Delta R$ falling below $1.42 \mathrm{hPa} \cdot \mathrm{s} \cdot \mathrm{L}^{-1}$ in asthmatic children with persistent wheeze [23].

\section{Future studies of clinical utility}

Studies are underway or being planned to examine the clinical utility of T-oscillometry in a number of paediatric respiratory conditions, including cystic fibrosis (CF), bronchopulmonary dysplasia (BPD), recurrent wheeze in preschool-aged children, sickle cell anaemia and neuromuscular conditions. CF lung disease begins in the small peripheral airways and is poorly detected by spirometry. Studies are underway to examine whether early disease is reflected by $X$ at $10 \mathrm{~Hz}\left(X_{10}\right)$ and $\Delta X$. Diffuse disease in small airways results in time-constant inhomogeneity and uneven ventilation distribution. These changes are detected by the lung clearance index, the test considered most sensitive to early CF lung disease. We contend that $X_{10}$ and $\Delta X$ will provide the same information without the need for $100 \%$ oxygen and in a much shorter measurement time. We have demonstrated that expiratory flow limitation is easily detected by plotting $X_{8}$ against tidal volume in adults with COPD [25], while $\Delta X$ reflects the improving homogeneity during inspiration. Studies are underway to determine whether similar changes will be seen in childhood survivors with BPD. Epidemiological studies show that $70 \%$ of preschool-aged children with recurrent wheeze do not develop persistent asthma. Current predictive indices do not accurately identify these children. Studies are planned to determine whether the presence of airway obstruction, as indicated by $\Delta R$, can identify children destined for persistent asthma.

The INCIRCLE CRC has made great advances in developing new modalities of oscillometry for use in infants and young children, and this has evoked interest from the lung function device industry. INCIRCLE has also developed co-operation with another CRC (the Global Lung function Initiative network (GLI)). The work is far from done but we are entering the exciting stage where we will determine where this test is clinically useful in diverse respiratory disorders. Watch this space for future developments!

Research groups interested in joining at this stage should contact the co-chairs Peter D. Sly (p.sly@uq.edu.au) or Zoltán Hantos (hantos.zoltan@med.u-szeged.hu), or the project manager Dorottya Czövek (czovek.dorottya@med. semmelweis-univ.hu).

Conflict of interest: P.D. Sly reports a peer-reviewed research grant from the National Health and Medical Research Council, Australia, during the conduct of the study. In addition, P.D. Sly is a named inventor on a patent owned by Telethon Kids Institute, Perth, Australia: "A method of measuring an acoustic impedance of a respiratory system and diagnosing a respiratory disease or disorder or monitoring treatment of same". This patent is licensed to Thorasys Canada. No royalty payments have been received. Z. Hantos is a named inventor on a patent owned by Telethon Kids Institute, Perth, Australia: "A method of measuring an acoustic impedance of a respiratory system and diagnosing a respiratory disease or disorder or monitoring treatment of same". This patent is licensed to Thorasys Canada. No royalty payments have been received.

Support statement: Funding was received from the European Respiratory Society, grant ERS CRC-2013-02 (INCIRCLE). Funding information for this article has been deposited with the Crossref Funder Registry.

\section{References}

1 Landrigan PJ, Sly JL, Ruchirawat M, et al. Health consequences of environmental exposures: changing global patterns of exposure and disease. Ann Glob Health 2016; 82: 10-19.

2 Sly PD, Carpenter DO, Van den Berg M, et al. Health consequences of environmental exposures: causal thinking in global environmental epidemiology. Ann Glob Health 2016; 82: 3-9.

3 Vos T, Flaxman AD, Naghavi M, et al. Years lived with disability (YLDs) for 1160 sequelae of 289 diseases and injuries 1990-2010: a systematic analysis for the Global Burden of Disease Study 2010. Lancet 2012; 380: 2163-2196.

4 Hopper JL, Hibbert ME, Macaskill GT, et al. Longitudinal analysis of lung function growth in healthy children and adolescents. J Appl Physiol 1991; 70: 770-777.

5 Owens L, Laing IA, Zhang G, et al. Airway function in infancy is linked to airflow measurements and respiratory symptoms from childhood into adulthood. Pediatr Pulmonol 2018; 53: 1082-1088.

6 Sly PD, Bush A. From the cradle to the grave: the early-life origins of chronic obstructive pulmonary disease. $A m J$ Respir Crit Care Med 2016; 193: 1-2. 
7 Morgan WJ, Stern DA, Sherrill DL, et al. Outcome of asthma and wheezing in the first 6 years of life: follow-up through adolescence. Am J Respir Crit Care Med 2005; 172: 1253-1258.

8 Robertson CF. Long-term outcome of childhood asthma. Med J Aust 2002; 177: Suppl., S42-S44.

9 Lange P, Celli B, Agusti A, et al. Lung-function trajectories leading to chronic obstructive pulmonary disease. N Engl J Med 2015; 373: 111-122.

10 Svanes C, Sunyer J, Plana E, et al. Early life origins of chronic obstructive pulmonary disease. Thorax 2010; 65: $14-20$.

11 Barnes PJ, Burney PG, Silverman EK, et al. Chronic obstructive pulmonary disease. Nat Rev Dis Primers 2015; 1: 15076.

12 Rosenfeld M, Allen J, Arets BH, et al. An official American Thoracic Society workshop report: optimal lung function tests for monitoring cystic fibrosis, bronchopulmonary dysplasia, and recurrent wheezing in children less than 6 years of age. Ann Am Thorac Soc 2013; 10: S1-S11.

13 Busi LE, Restuccia S, Tourres R, et al. Assessing bronchodilator response in preschool children using spirometry. Thorax 2017; 72: 367-372.

14 Joseph-Bowen J, de Klerk NH, Firth MJ, et al. Lung function, bronchial responsiveness, and asthma in a community cohort of 6-year-old children. Am J Respir Crit Care Med 2004; 169: 850-854.

15 Brightling C, Genton C, Bill W, et al. ERS Clinical Research Collaborations: underpinning research excellence. Eur Respir J 2018; 52: 1801534.

16 Gangell CL, Horak F Jr, Patterson HJ, et al. Respiratory impedance in children with cystic fibrosis using forced oscillations in clinic. Eur Respir J 2007; 30: 892-897.

17 Ramsey KA, Ranganathan SC, Gangell CL, et al. Impact of lung disease on respiratory impedance in young children with cystic fibrosis. Eur Respir J 2015; 46: 1672-1679.

18 Thamrin C, Gangell CL, Udomittipong K, et al. Assessment of bronchodilator responsiveness in preschool children using forced oscillations. Thorax 2007; 62: 814-819.

19 Hantos Z, Czövek D, Gyurkovits Z, et al. Assessment of respiratory mechanics with forced oscillations in healthy newborns. Pediatr Pulmonol 2015; 50: 344-352.

20 Gray D, Czövek D, Smith E, et al. Respiratory impedance in healthy unsedated South African infants: effects of maternal smoking. Respirology 2015; 20: 467-473.

21 Shackleton C, Czövek D, Grimwood K, et al. Defining "healthy" in preschool-aged children for forced oscillation technique reference equations. Respirology 2018; 23: 406-413.

22 Gray D, Willemse L, Visagie A, et al. Determinants of early-life lung function in African infants. Thorax 2017; 72 : $445-450$.

23 Czövek D, Shackleton C, Hantos Z, et al. Tidal changes in respiratory resistance are sensitive indicators of airway obstruction in children. Thorax 2016; 71: 907-915.

24 Shackleton C. Improving the clinical utility of the forced oscillation technique in preschool-aged children. $\mathrm{PhD}$ Thesis. Brisbane, The University of Queensland, 2018. Available from: https://doi.org/10.14264/uql.2018.481

25 Lorx A, Czövek D, Gingl Z, et al. Airway dynamics in COPD patients by within-breath impedance tracking: effects of continuous positive airway pressure. Eur Respir J 2017; 49: 1601270. 